Vol. 7, No. 1, 2017

\title{
ESTIMATION METHOD FOR A SET OF SOLUTIONS TO INTERVAL SYSTEM OF LINEAR ALGEBRAIC EQUATIONS WITH OPTIMIZED "SATURATED BLOCK" SELECTION PROCEDURE
}

\author{
Mykola Dyvak, Iryna Oliynyk \\ Ternopil National Economic University, UKRAINE \\ mdy@tneu.edu.ua,ois@tneu.edu.ua
}

CC Dyvak M., Oliynyk I., 2017

\begin{abstract}
The paper substantiates the necessity of applying a new method for the formation of a set of basic equations in the problem of localizing solutions to an interval system of linear algebraic equations (ISLAE) on the basis of a "saturated block". The method is based on solving the problem of optimization. Th e minimization of the maximal prediction error by using interval models the parameters of which belong to the localization area of ISLAE solutions is chosen as a criterion. A comparative analysis of the effectiveness of the proposed method for finding the optimal "saturated block" and the methods of stochastic search, in particular with linear tactics and by best attempt is conducted. A significant advantage of the proposed method by the criterion of minimum computational complexity is shown.

Key words: identification, interval analysis, solutions localization, "saturated block" of interval system of linear algebraic equations, stochastic method.
\end{abstract}

\section{Introduction}

The problem of identifying parameters of static systems' models that is based on interval data is formulated as the problem of solving an interval system of linear algebraic equations (ISLAE) [1-3]. Unlike a regular system of linear algebraic equations, solutions to ISLAE are a set of parameter estimates of a static system's model. For the model to be used conveniently in applied research, instead of "exact" ISLAE solution some estimate of the solutions set is widely employed, e.g. in the form of multidimensional parallelotope or ellipsoid. A method that involves selection of a "saturated block", i.e. $m$ interval equations (such equations are called basic equations) from ISLAE with $m$ unknown values is distinguished among the existing methods for the estimation of an ISLAE solution area.

The localization area of solutions to the whole ISLAE [1-3] is built on the basis of the basic equations selected. The detailed algorithm of the above mentioned method is described in paper [4]. It is noted that selection of the "saturated block" which would provide optimal estimates of the parameters of a static system's model as a set is a computational problem of polynomial complexity [1-4].

It is obvious that the notion of optimality of a set of model parameter estimates is defined by the criterion of "saturated block" selection. Usually, this criterion minimizes the size of a parameter estimates area. However, such selection of a "saturated block" does not provide optimal prediction properties of the static system's model. To select a "saturated block", papers [5-8] propose to apply the criterion of minimization of the maximal prediction error of the model instead of using the methods of random search. To reduce the computational complexity related to realization of this approach, it is proposed to use the methods of the theory of design of optimal sequential experiments [9-10].

The comparison results of the methods of theory of design of optimal sequential experiments with those of stochastic search, in terms of their application, are represented in the paper.

\section{Statement of the problem}

Let us consider a static (non-inertial) system in which the dependence between "output" and "inputs" is represented in the form of the following algebrical equation:

$$
y_{0}=\beta_{1} \cdot \phi_{1}(\dot{x})+\mathrm{K}+\beta_{m} \cdot \phi_{m}\left(\dot{x}^{\prime}\right),
$$

where $y_{0}$ is the uknown true value of the system "output"; $\quad \stackrel{r}{x \in R^{m}}$ is the vector of input variables; ${ }^{\prime}=\left(\beta_{1}, \mathrm{~K}, \beta_{m}\right)^{T}$ is the vector of unknown parameters, $\dot{\phi}^{T}(\stackrel{r}{x})=\left(\phi_{1}(\stackrel{r}{x}), \mathrm{K}, \phi_{m}(\stackrel{r}{x})\right)^{T}$ is the vector of known basis functions.

To estimate the values of parameters, we use the experiment results in the form of matrix of input variables $X$ and vector of output variable values with known limit values of errors:

$$
X=\left(\begin{array}{ll}
x_{11} \mathrm{~L} & x_{1 n} \\
\mathrm{M} & \\
x_{i 1} \mathrm{~L} & x_{i n} \\
\mathrm{M} & \\
x_{N 1} \mathrm{~L} & x_{N n}
\end{array}\right) ;\left[\begin{array}{r}
\mathrm{r} \\
\mathrm{r}
\end{array}\right)=\left(\begin{array}{l}
{\left[y_{1}^{-} ; y_{1}^{+}\right]} \\
\mathrm{M} \\
{\left[y_{i}^{-} ; y_{i}^{+}\right]} \\
\mathrm{M} \\
{\left[y_{N}^{-} ; y_{N}^{+}\right]}
\end{array}\right),
$$

where $0,5\left(y^{+}(\stackrel{r}{x})-y^{-}(\stackrel{r}{x})\right)=\Delta(\stackrel{r}{x})$ is some known limit value of the measurement error. 
In this case, the following conditions are met:

$$
y_{i}^{-} \leq y_{0 i} \leq y_{i}^{+}, i=1, \mathrm{~K}, N,
$$

Based on conditions (1) and (3), ISLAE is obtained for the estimation of the vector of unknown parameters $\beta$ :

$$
\left\{\begin{array}{l}
y_{1}^{-} \leq b_{1} \phi_{1}\left(\stackrel{r}{x_{1}}\right)+\mathrm{K}+b_{m} \phi_{m}\left(\stackrel{r}{x_{1}}\right) \leq y_{1}^{+} \\
\mathrm{M} \\
y_{i}^{-} \leq b_{1} \phi_{1}\left(\stackrel{r}{x}_{i}\right)+\mathrm{K}+b_{m} \phi_{m}\left(\stackrel{r}{x_{i}}\right) \leq y_{i}^{+} \\
\mathrm{M} \\
y_{N}^{-} \leq b_{1} \phi_{1}\left({ }^{\mathrm{r}} x_{N}\right)+\mathrm{K}+b_{m} \phi_{m}\left(x_{N}\right) \leq y_{N}^{+}
\end{array} .\right.
$$

The set $\Omega$ is the solutions to ISLAE (4):

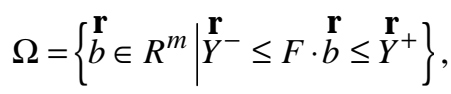

where $F=\left\{\phi_{j}^{\mathrm{r}}\left(x_{i}^{\mathrm{r}}\right), i=1, \mathrm{~K}, N, j=1, \mathrm{~K}, m\right\}$ is the known matrix of basis functions values, $\stackrel{r}{Y}^{-}=\left\{y_{i}^{-}, i=1, \mathrm{~K}, N\right\}$ and $\stackrel{r}{Y^{+}}=\left\{y_{i}^{+}, i=1, \mathrm{~K}, N\right\}$ are the vectors formed from upper and lower bounds of the intervals $\left[y_{i}^{-} ; y_{i}^{+}\right]$.

If ISLAE (4) is non-degerate, then geometrically its area of solutions is a convex polyhedron [1-3]. However, instead of using the solution area in form (5), it is expedient to use its estimations in the form of multidimensional rectangular parallelepipeds or ellipsoids. The noted methods for the estimation of ISLAE solutions area are not always suitable for solving the applied problems of modeling due to low accuracy of the first one and high level of complexity of the second one.

Recently, the methods of estimating the ISLAE solution area, which are based on the analysis of "informativeness" of each ISLAE interval equation, have become widely used. As known from [1-3], on the one hand there may be equations in ISLAE that do not define the solution area, and on the other hand there may be interval equations that do not change significantly the configuration and size of the solution area. One of such methods is described in papers [11-13]. It consists in defining a solution area configuration at the initial stage. For this, it is necessary to select $m$ equations from ISLAE and then change the size of the given configuration area taking into account the other equations from ISLAE.

Example. Let ISLAE be in the following form:

$$
\left\{\begin{array}{l}
9,946 \leq 6 b_{0}-0,279 b_{1} \leq 12,178 \\
14,313 \leq 7 b_{0}+0,657 b_{1} \leq 17,507 \\
17,33 \leq 9 b_{0}+0,412 b_{1} \leq 21,177 \\
4,042 \leq b_{0}+0,841 b_{1} \leq 4,947 \\
5,846 \leq 3 b_{0}+0,141 b_{1} \leq 7,13
\end{array},\right.
$$

Let us select a "saturated block", for example, a system consisting of the first and second equations from the given ISLAE:

$$
\left\{\begin{array}{l}
9,946 \leq 6 b_{0}-0,279 b_{1} \leq 12,178 \\
14,313 \leq 7 b_{0}+0,657 b_{1} \leq 17,507
\end{array} .\right.
$$

According to the algorithm of realization of the method of "saturated block" selection, let us add, for example, the fifth equation:

$$
5,846 \leq 3 b_{0}+0,141 b_{1} \leq 7,13,
$$

The result of the algorithm application is shown in Fig. 1.

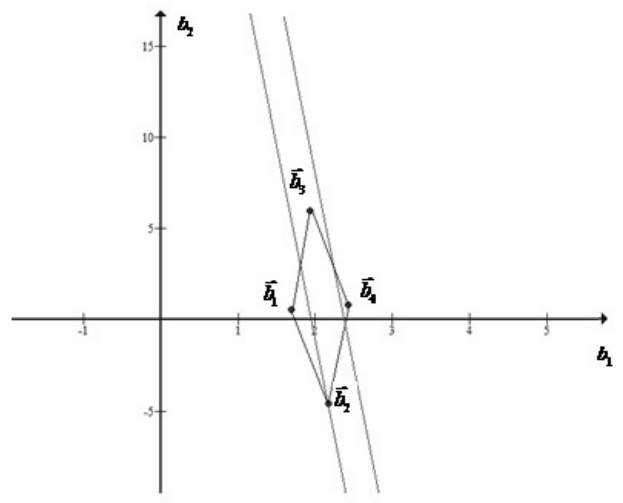

Fig. 1. Illustration of solutions to interval equations (7) and (8).

In another case, let us choose a "saturated block" formed from the first and third equations from ISLAE (6):

$$
\left\{\begin{array}{l}
9,946 \leq 6 b_{0}-0,279 b_{1} \leq 12,178 \\
17,33 \leq 9 b_{0}+0,412 b_{1} \leq 21,177
\end{array}\right. \text {. }
$$

And add equation (8) (Fig. 2).

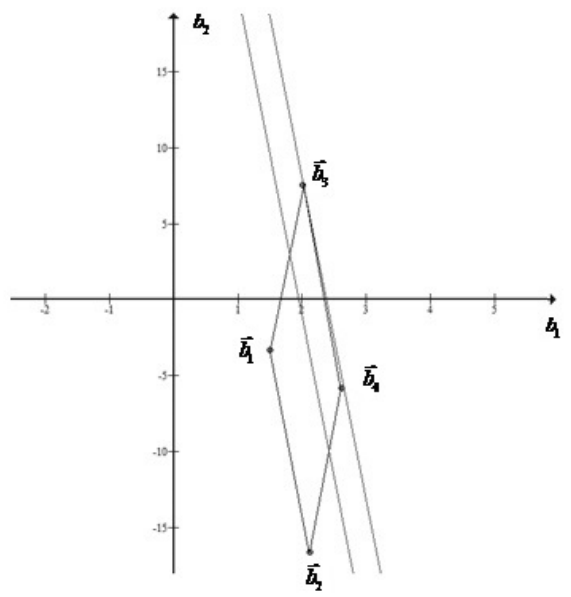

Fig. 2. Illustration of solutions to interval equations (8) and (9).

As we can see in the Figures, system (7) defines the configuration in the form of parallelogram. Adding equation (8) does not change the size of the area, and adding equation (9) reduces the area of solutions. Thus, 
we can see that the most difficult step is selection of basic equations that form a "saturated block".

Papers [11-13] propose the minimization of maximal prediction error in the range of values of input variables $x \in \chi$ as the criterion of selecting the optimal "saturated block":

$$
\begin{aligned}
& \Delta_{\text {max }}\left(F_{m}\right)=\max _{x \in \chi}\left\{2 \cdot \sum_{j=1}^{m}\left|\alpha_{j}(\stackrel{r}{x}) \cdot \Delta_{j}\right|\right\} \stackrel{F_{m}}{\longrightarrow} \min , \\
& \stackrel{r}{\alpha}^{T}(\stackrel{r}{x})=\stackrel{r}{\varphi}^{T}(\stackrel{r}{x}) \cdot F_{m}^{-1} .
\end{aligned}
$$

If all the equations in ISLAE (4) are numbered: $1, \ldots, i, \ldots, N$, respectively, then each equation in the "saturated block" has a unique number $n_{j}$, where $n$ is number of the equation from system (4), $j=1, \ldots, m$ is its index number in the "saturated block". Then expression (10) can be rewritten as:

$$
F_{m}^{-1}=\psi\left(n_{1}, n_{2}, \ldots, n_{j}, \ldots, n_{m}\right),
$$

And we obtain:

$$
\begin{aligned}
& \Delta_{\max }\left(n_{1}, \ldots, n_{m}\right)= \\
& \quad=\max _{x \in \chi}\left\{2 \cdot \sum_{j=1}^{m}\left|\alpha_{j}\left(x^{r}\right) \cdot \Delta_{j}\right|\right\} \stackrel{\psi\left(n_{1}, \ldots, n_{m}\right)}{\longrightarrow} \min , \\
& \stackrel{r}{\alpha}^{r}(\stackrel{r}{r})=\varphi^{T}(\stackrel{r}{x}) \cdot \psi\left(n_{1}, \ldots, n_{m}\right) .
\end{aligned}
$$

Expression (12) provides the minimization of maximal prediction error of the interval model among all possible "saturated blocks" selected from ISLAE (4).

\section{Methods of solving the problem of optimal "saturated block" selection}

Full combinatorial enumeration [1-3] and stochastic search [5-8] are distinguished among the known methods of solving the problem of optimal "saturated block" selection.

Taking into account the fact that combinatorial enumeration of all possible "saturated blocks" is extremely consumable from the computational point of view, let us consider the methods of stochastic search in more detail.

The random search method with linear tactics. This method is developed using two operators: random step and repetition of the previous step [5-8]. Using each of them may lead to one of two results: the value of a minimized function either gets reduced or remains unchanged. Depending on the result, one or the other operator is used.

The random search algorithm with linear tactics is based on the following obvious assumption concerning the object of optimization: probability of a successful step in the direction that was successful in the previous iteration will be higher than the probability of a successful step chosen randomly. The linearity of the tactics of this procedure consists in simulation of linear behavior, i.e in the exact repetition of a successful step [5-8].
Let us interpret the algorithm of random search method for the formation of optimal "saturated block" in the problem of localization of ISLAE solutions.

\begin{tabular}{|c|c|c|c|c|c|c|c|}
\hline & \multicolumn{7}{|c|}{ Number of second equation of "saturated block", $\mathrm{n}_{2}$} \\
\hline \multirow{7}{*}{ 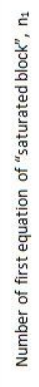 } & $i / i$ & 1 & 2 & 3 & 4 & 5 & 6 \\
\hline & 1 & $\infty$ & $\Delta_{\operatorname{mat}}(\psi(1,2))$ & $\Delta_{\operatorname{man}}(\psi(1,3))$ & $\Delta_{\operatorname{man}}(\psi(1,4))$ & $\Delta_{\max }(\psi(1,5))$ & $\Delta_{\sin }(\psi(1,6))$ \\
\hline & 2 & & $\infty$ & $\Delta_{\operatorname{man}}(\psi(2,3))$ & $\Delta_{\max }(\psi(2,4))$ & $\Delta_{\max }(\psi(2,5))$ & $\Delta_{\max }(\psi(2,6))$ \\
\hline & 3 & & & $\infty$ & $\Delta_{\operatorname{man}}(\psi(3,4))$ & $\Delta_{\text {att }}(\psi(3,5))$ & $\Delta_{\max }(\psi(3,6))$ \\
\hline & 4 & & & & $\infty$ & $\Delta_{\max }(\psi(4,5))$ & $\Delta_{\max }(\psi(4,6))$ \\
\hline & 5 & & & & & $\infty$ & $\Delta_{\operatorname{man}}(\psi(5,6))$ \\
\hline & 6 & & & & & & $\infty$ \\
\hline
\end{tabular}

Let us illustrate the algorithm of the random search method with linear tactics for the two-dimensional case $(m=2, N=6)$. The space of the objective function values for all possible "saturated blocks" is represented in the table in Fig. 3.

Fig. 3. Table of objective function values for all possible "saturated blocks" in the case $m=2, N=6$.

Let us select an initial "saturated block", for example, $\psi(2,3)$ (circled in Fig. 4), and for the block define the values of the objective function $\Delta_{\max }(\psi(2,3))$. The size of the step is considered to be the difference between the equation numbers in the "saturated block". For example, if the initial set of equations is $\left(n_{1} ; n_{2}\right)$, length of the step equals 1 , then the following directions are obtained: $\left(n_{1}-1 ; n_{2}\right)$, $\left(n_{1}+1 ; n_{2}\right), \quad\left(n_{1} ; n_{2}-1\right), \quad\left(n_{1} ; n_{2}+1\right)$. Let $\left(n_{1} ; n_{2}+1\right)$ be chosen as a random direction. Therefore, the next "saturated block" is $\psi(2,4)$.

Let us define the value of the objective function $\Delta_{\max }(\psi(2,4))$ and check the condition $\Delta_{\max }(\psi(2,4))<\Delta_{\max }(\psi(2,3))$. If the condition is met, then the calculations are continued in the same direction. Let us suppose that $\Delta_{\max }(\psi(2,6))<\Delta_{\max }(\psi(2,5))$. In this case, according to the scheme of random search algorithm, it is necessary to change the direction of linear tactics. Among the directions available, we select $\left(n_{1}+1 ; n_{2}\right)$. The resulting "saturated block" is $\psi(3,5)$. Let us determine the value of the objective function $\Delta_{\max }(\psi(3,5))$ and check the condition $\Delta_{\max }(\psi(3,5))<\Delta_{\max }(\psi(2,5))$. If the condition is met, the direction is considered successful. Further) calculations are carried out according to the direction and by analogy with the previous steps. Fig. 4 illustrates the above-described sequence of calculations implemented in the space of ISLAE equation numbers.

The method of random search by the best attempt. This method is based on two basic concepts: the 
direction of calculation and step [5-8]. Firstly, let us choose the size of the step and find the values of the objective function for all the possible directions, and then select the minimal one. From this point, again, let us carry out the calculations in all the directions according to the size of the step chosen. By analogy with the previous iterations, among the selected values, the minimal one is chosen.

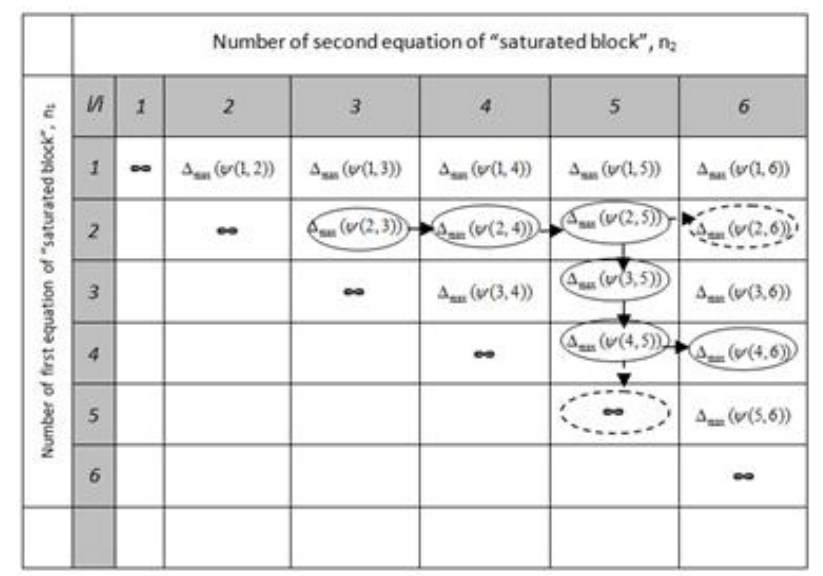

Fig. 4. Illustration of random search strategy with linear tactics.

Let us interpret the algorithm of random search by the best attempt for forming the optimal "saturated block" in the problem of localization of ISLAE solutions.

Let us illustrate the algorithm of the above method for the two-dimensional case from the table in Fig. 3. As an example, let $\psi(2,4)$ be selected the initial "saturated block"(circled in Fig. 5). Then, for this block, we define the value of the objective function $\Delta_{\max }(\psi(2,4))$. As noted above, the size of the step is considered to be the difference between the equation numbers in the "saturated block". For example, if the initial set of equations is $\left(n_{1} ; n_{2}\right)$, length of the step is equal to 1 , then, we obtain the following directions: $\left(n_{1}-1 ; n_{2}\right)$, $\left(n_{1}+1 ; n_{2}\right),\left(n_{1} ; n_{2}-1\right),\left(n_{1} ; n_{2}+1\right)$. If the step length equals 1 , then, based on the table in Fig. 3 , the following "saturated blocks" to be studied are $\psi(2,3), \psi(1,4)$, $\psi(2,5), \psi(3,4)$ (circled by a dotted line in Fig. 5). The values of the objective functions are defined for the blocks $\Delta_{\max }(\psi(2,3)), \quad \Delta_{\max }(\psi(1,4)), \quad \Delta_{\max }(\psi(2,5))$, and $\Delta_{\max }(\psi(3,4))$. Among the values obtained, let us find the minimal one, for example, $\Delta_{\max }(\psi(2,5))$. Accordingly, the further calculations are carried out concerning the "saturated block» $\psi(2,5)$. By analogy with the previous step, the value of the objective function is defined for the blocks $\Delta_{\max }(\psi(1,5)), \Delta_{\max }(\psi(3,5))$ and $\Delta_{\max }(\psi(2,6))$. Let us find the minimum of them, for example, for the "saturated block" $\psi(3,5)$. The next calculations must be carried out by analogy with the previous steps. The above-described sequence of calculations realizated in the space of ISLAE equation numbers is represented in Fig. 5.

The method of forming a set of basic equations in the problem of localization of ISLAE solutions. Now, let us consider the proposed method of directed enumeration of "saturated blocks" developed according to the procedure of $I_{G}$-optimal experiment design. The essence of the method consists in selecting some "saturated block", obtaining the corridor of interval models and analyzing the predictive properties of these models that is the basis for planning the way of forming the next "saturated block".

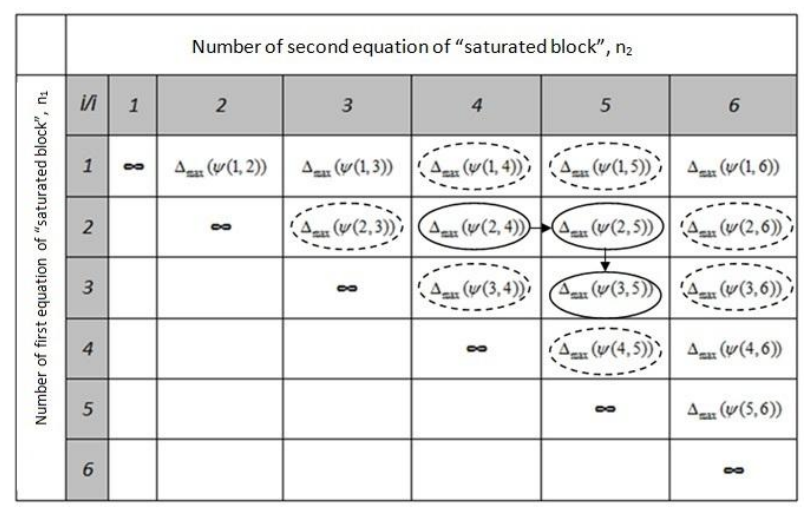

Fig. 5. Illustration of random search strategy by the best attempt.

Thus, let the structure of mathematical model of static system be defined by expression (1) with unknown parameters, interval data (2) be given and ISLAE be formed in the form of (4).

Let us choose a "saturated block" from ISLAE randomly, calculate its solution area and build the corridor of prediction by interval models:

$$
\begin{aligned}
& {[\mathcal{y}(\dot{x})]=} \\
& \left.\left.=\left[\dot{\varphi}^{T}(\underset{x}{\mathrm{r}}) \cdot \frac{\mathrm{r}}{b}-\frac{1}{2} \cdot \Delta\right)_{y(x)}^{\underset{r}{r}} ; \dot{\varphi}^{T}(\dot{r}) \cdot \frac{r}{b}+\frac{1}{2} \cdot \Delta\right) \underset{y}{r}(x)\right]
\end{aligned}
$$

Now, by analogy with the procedure of sequential $I_{G}$-optimal experiment design, it is necessary to calculate the vector ${ }_{x}$ max , which provides the maximal prediction error in the area of the experiment:

$$
\begin{aligned}
& x^{r_{m a x}}=\arg \underset{x_{i}=1, \ldots, N}{\max }\left\{2 \cdot \sum_{j=1}^{m}\left|\alpha_{j}\left(x_{i}\right) \cdot \Delta_{j}\right|, x_{i}^{r}, i=1, \ldots, N\right\},
\end{aligned}
$$

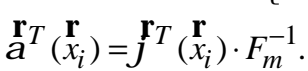

It should be noted that procedure (14) is simple as it is implemented for the finite set of points $x_{i}, i=1, \ldots, N$. The vector obtained by expression (14) is a vector of values of input variables. This vector defines a certain interval equation in ISLAE (4). In accordance with the procedure of sequential 
$I_{G}$-optimal experiment design, it is exactly this point at which the next measurement should be conducted.

Papers [11-13] prove that if the vector ${ }_{x}^{r}$ max coincides with the vector of values of the input variables of one of the interval equations of the ISLAE "saturated block", then it specifies the point with the minimal value of prediction error. Hence, it is advisable to replace one of the interval equations in the current "saturated block" by the interval equation of ISLAE with the vector of input variables values ${ }_{x}$ max defined by expression (14). Thus, by analogy with the procedure of sequential $I_{G}$-optimal experiment design, we "simulate" the procedure of additional measurement at the point ${ }^{r_{m a x}}$ with the maximal prediction error of interval model. This procedure is conducted for each interval equation in the "saturated block" resulting in obtaining $p$ $(p=1, \ldots, m)$ new "saturated blocks".

As a result, $m$ values of maximal errors for the corresponding interval models are obtained for each of the $m$ "saturated blocks":

$$
\begin{aligned}
& \Delta_{\max }^{p}=\max _{x_{i}, i=1, \ldots, N}\left\{2 \cdot \sum_{j=1}^{m}\left|\alpha_{j p}\left(r_{i}^{r}\right) \cdot \Delta_{j}\right|\right\}, \\
& \alpha_{p}^{r_{T}}\left(x_{i}\right)=\stackrel{r}{\varphi}^{T}\left(x_{i}\right) \cdot F_{m}^{-1}(p), p=1, \ldots, m
\end{aligned}
$$

where $p$ is the index which indicates a "saturated block" number, $F_{m}(p)$ is the matrix of basic functions values for the $p$-th block, $\alpha_{j p}\left(x_{i}\right)$ is the $i$-th component of vector $\stackrel{\alpha}{\alpha}$ calculated for the $p$-th "saturated block".

It is obvious that for the optimal "saturated block" to be selected at this step, instead of using complex computational procedure (10), it is sufficient to select from the $m$ "saturated blocks" the one that provides the least value of sequence (15):

$$
F_{m}{ }^{o p t}=\underset{p=1, \ldots, m}{\arg \min }\left\{\Delta^{p}{ }_{\max }, p=1, \ldots, m\right\},
$$

Using procedure (12), we obtain $\stackrel{r}{x}^{\max }$ - the vector for which the prediction error of the interval model is maximal. This interval model is defined by the solution to "saturated block" (16). The iterations then continue until the "saturated block" whose equations can be replaced without leading to a decrease in the maximum prediction error by interval models is obtained.

As we can see from expression (12), the objective function in the problem of "saturated block" selection is discrete, since its value is defined by a specific set of interval equations. It is also obvious that this function is not unimodal.

\section{Example of estimating the time complexity of the methods}

Let us analyze the effectiveness of the proposed method on an example. Let the equation for developing an interval model have the following general form:

$$
y(x)=b_{0} \cdot x+b_{1} \cdot \ln (x),
$$

The parameters of the model are defined from the set of "experimental" data represented in Fig. 6.

\begin{tabular}{|c|c|c|c|c|c|c|c|}
\hline $\mathrm{i}$ & $x_{i}$ & $y_{i}^{-}$ & $y_{i}^{+}$ & $\mathrm{i}$ & $x_{i}$ & $y_{i}^{-}$ & $y_{i}^{+}$ \\
\hline 1 & 1 & 1,7 & 2,1 & 11 & 32 & 73,294 & 89,559 \\
\hline 2 & 5 & 16,181 & 19,791 & 12 & 33 & 75,058 & 91,755 \\
\hline 3 & 9 & 26,105 & 31,902 & 13 & 35 & 78,901 & 96,456 \\
\hline 4 & 10 & 28,332 & 34,634 & 14 & 37 & 82,855 & 101,266 \\
\hline 5 & 14 & 37,14 & 45,379 & 15 & 40 & 88,62 & 108,309 \\
\hline 6 & 16 & 41,211 & 50,384 & 16 & 42 & 92,353 & 112,89 \\
\hline 7 & 19 & 47,492 & 58,037 & 17 & 50 & 107,594 & 131,506 \\
\hline 8 & 23 & 55,471 & 67,806 & 18 & 57 & 120,705 & 147,548 \\
\hline 9 & 28 & 65,313 & 79,845 & 19 & 59 & 124,606 & 152,283 \\
\hline 10 & 29 & 67,282 & 82,25 & 20 & 60 & 126,429 & 154,523 \\
\hline
\end{tabular}

Fig. 6. Table of "experimental" data.

Then, the interval system for finding the coefficients of the model is as follows:

$$
\left\{\begin{array}{l}
1,7 \leq b_{0} \leq 2,1 \\
16,181 \leq 5 b_{0}+b_{1} \leq 19,971 \\
26,105 \leq 9 b_{0}+2,197225 b_{1} \leq 31,902 \\
28,332 \leq 10 b_{0}+2,302585 b_{1} \leq 34,634 \\
37,14 \leq 14 b_{0}+2,639057 b_{1} \leq 45,379 \\
41,211 \leq 16 b_{0}+2,772589 b_{1} \leq 50,384 \\
47,492 \leq 19 b_{0}+2,944439 b_{1} \leq 58,037 \\
55,471 \leq 23 b_{0}+3,135494 b_{1} \leq 67,806 \\
65,313 \leq 28 b_{0}+3,332205 b_{1} \leq 79,845 \\
67,282 \leq 29 b_{0}+3,367296 b_{1} \leq 82,25 \\
73,294 \leq 32 b_{0}+3,465736 b_{1} \leq 89,559 \\
75,058 \leq 33 b_{0}+3,496508 b_{1} \leq 91,755 \\
78,901 \leq 35 b_{0}+3,555348 b_{1} \leq 96,456 \\
82,855 \leq 37 b_{0}+3,610918 b_{1} \leq 101,266 \\
88,62 \leq 40 b_{0}+3,688879 b_{1} \leq 108,309 \\
92,353 \leq 42 b_{0}+3,73767 b_{1} \leq 112,89 \\
107,594 \leq 50 b_{0}+3,912023 b_{1} \leq 131,506 \\
120,705 \leq 57 b_{0}+4,043051 b_{1} \leq 147,548 \\
124,606 \leq 59 b_{0}+4,077537 b_{1} \leq 152,283 \\
126,429 \leq 60 b_{0}+4,094345 b_{1} \leq 154,523
\end{array} .\right.
$$

Let us number the equations in system (18). Accordingly, the interval equation $1,7 \leq b_{0} \leq 2,1$ is numbered 1 , and the interval equation $126,429 \leq 60 b_{0}+4,094345 b_{1} \leq 154,523$ is numbered 20 .

According to expression (17), the values of the objective function are calculated and introduced into the table in Fig. 7. In the table (see Fig. 7), $N_{1}$ stands for the 
number of the first equation in the "saturated block", $N_{2}$ is the number of the second equation.

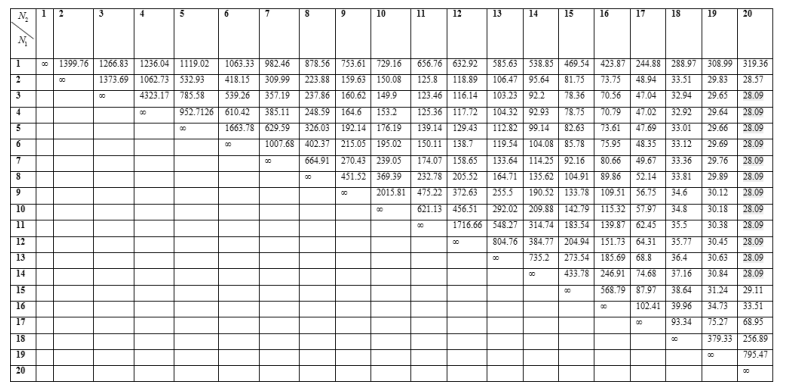

Fig. 7. Table of values of the objective function calculated using the method of full combinatorial enumeration..

As we can see, the objective function is discrete and has a great number of local minimums. Let us apply the algorithm of random search method with linear tactics to the example described above. At the first stage of calculations we select a "saturated block", for example, $\psi(7,13)$ :

$$
\left\{\begin{array}{l}
47,492 \leq 19 b_{0}+2,944439 b_{1} \leq 58,037 \\
78,901 \leq 35 b_{0}+3,555348 b_{1} \leq 96,456
\end{array} .\right.
$$

The maximal prediction error of the interval model developed for this "saturated block" is 133,64.

Let us generate a random direction - $\left(n_{1}-1 ; n_{2}\right)$. Thus, we move to the point that corresponds to the "saturated block" $\psi(6,13)$. The maximal prediction error of the interval model for this "saturated block" is 119,54 .

Using the algorithm for the method of random search with linear tactics resulted in decreasing the value of the objective function. That is why the chosen direction is considered sucessfull. In the next iterations, the "saturated blocks" $\psi(5,13), \quad \psi(4,13)$ and $\psi(3,13), \quad \psi(2,13)$, respectively, are examined by turns. The results obtained while examining the "saturated block" $\psi(2,13)$ do not meet the conditions of the method. Therefore, in accordance with the algorithm of random search, it is necessary to generate a new direction of linear tactics with regard to the "saturated block" $\psi(3,13)$.

Let us generate the random direction $\left(n_{1}+1 ; n_{2}\right)$ and examine the "saturated block" $\psi(3,14)$. For the block above, the value of the function $\Delta_{\max }(\psi(3,14))$ is equal to 92, 2. Now let us check the condition $\Delta_{\max }(\psi(3,14))<\Delta_{\max }(\psi(3,13))$. As the condition is met, the chosen direction is considered successful. By analogy with the previous steps, let us sequentially study the "saturated blocks" $\psi(3,15), \psi(3,16), \psi(3,17), \psi(3,18)$, $\psi(3,19)$ and $\psi(3,20)$, respectively.

The "saturated block" $\psi(3,20)$ with the maximal prediction error 28,09 is considered the optimal "saturated block". It provides a numeric value of the objective function as a minimum of maximal error for interval models is 28,09 .

The scheme of calculations based on the fragment of the table in Fig. 7 with appropriate marking is shown in Fig. 8.

\begin{tabular}{|l|l|l|l|l|l|l|l|l|l|}
\hline$N_{2}$ & $\mathbf{1 2}$ & $\mathbf{1 3}$ & $\mathbf{1 4}$ & $\mathbf{1 5}$ & $\mathbf{1 6}$ & $\mathbf{1 7}$ & $\mathbf{1 8}$ & $\mathbf{1 9}$ & $\mathbf{2 0}$ \\
\hline $\mathbf{l}$ & 632.92 & 585.63 & 538.85 & 469.54 & 423.87 & 244.88 & 288.97 & 308.99 & 319.36 \\
\hline $\mathbf{2}$ & 118.89 & $: 106.47$ & 95.64 & 81.75 & 73.75 & 48.94 & 33.51 & 29.83 & 28.57 \\
\hline $\mathbf{3}$ & 116.14 & 103.23 & 92.2 & 78.36 & 70.56 & 47.04 & 32.94 & 29.65 & 28.09 \\
\hline $\mathbf{4}$ & 117.72 & 104.32 & 92.93 & 78.75 & 70.79 & 47.02 & 32.92 & 29.64 & 28.09 \\
\hline $\mathbf{5}$ & 129.43 & 112.82 & 99.14 & 82.63 & 73.61 & 47.69 & 33.01 & 29.66 & 28.09 \\
\hline $\mathbf{6}$ & 138.7 & 119.54 & 104.08 & 85.78 & 75.95 & 48.35 & 33.12 & 29.69 & 28.09 \\
\hline $\mathbf{7}$ & 158.65 & 133.64 & 114.25 & 92.16 & 80.66 & 49.67 & 33.36 & 29.76 & 28.09 \\
\hline $\mathbf{8}$ & 205.52 & 164.71 & 135.62 & 104.91 & 89.86 & 52.14 & 33.81 & 29.89 & 28.09 \\
\hline
\end{tabular}

Fig. 8. Scheme of calculating the minimal value of the objective function using random search with linear tactics based on the fragment of the table in Fig. 7.

Let us apply the algorithm of random search by the best attempt method to the example under consideration. At the first stage of calculation, the "saturated block" $\psi(7,13)(19)$ is selected.

The maximal prediction error of the interval model developed for this "saturated block" is 133,64 . The value of the step is 1 . Let us define all possible calculation directions for the two-dimensional case proposed: $\left(n_{1}-1 ; n_{2}\right),\left(n_{1}+1 ; n_{2}\right),\left(n_{1} ; n_{2}-1\right),\left(n_{1} ; n_{2}+1\right)$.

Therefore, it is necessary to analyze the value of the objective function for the "saturated blocks" $\psi(7,12)$, $\psi(7,14), \psi(6,13)$, and $\psi(8,13)$ concerning the initially selected "saturated block" $\psi(7,13)$. This results in the following values: $\Delta_{\max }(\psi(7,12))=158,65$; $\Delta_{\max }(\psi(7,14))=114,25 ; \quad \Delta_{\max }(\psi(6,13))=119,54 ;$ $\Delta_{\max }(\psi(8,13))=164,71$. The minimal value among the obtained ones corresponds to the "saturated block" $\psi(8,13)$. Therefore, the calculations in the next iteration are carried out concerning to the block, without changing a step value and characteristics of the directions.

Thus, realizing the algorithm of the method of random search by the best attempt, we obtained a sequential reduction of the objective function value in each iteration and found a local extremum for the "saturated block" $\psi(7,20)$.

Such a "saturated block" provides the numeric value of objective function 28,09 as a minimum of maximal error for interval models. The scheme of calculations based on the fragment of the table in Fig.7 with appropriate marking is shown in Fig. 9.

Now, let us apply the developed method for the selection of optimal "saturated block" for ISLAE (18). At the first step of algorithm realization, the "saturated block" $\psi(7,13)$ in the form of $(19)$ is selected. 


\begin{tabular}{|c|c|c|c|c|c|c|c|c|c|}
\hline$N_{N_{1}}^{N_{2}}$ & 12 & 13 & 14 & 15 & 16 & 17 & 18 & 19 & 20 \\
\hline 1 & 32.92 & 585.63 & 538.85 & 469.54 & 423.87 & 244.88 & 288.97 & 308.99 & 319.36 \\
\hline 2 & 118.89 & 106.47 & 95.64 & 81.75 & 73.75 & 48.94 & 33.51 & 29.83 & 28.57 \\
\hline 3 & 116.14 & 103.23 & 92.2 & 78.36 & 70.56 & 47.04 & 32.94 & 29.65 & 28.09 \\
\hline 4 & 117.72 & 104.32 & 92.93 & 78.75 & 70.79 & 47.02 & 32.92 & 29.64 & 28.09 \\
\hline 5 & 129.43 & 112.82 & 99.14 & 82.63 & 73.61 & 47.69 & 33.01 & 29.66 & 28.09 \\
\hline 6 & 138.7 & 119.54 & 104.08 & 85.78 & 75.95 & 48.35 & 33.12 & 29.69 & 28.09 \\
\hline 7 & 158.65 & 133.64 & 114.25 & 92.16 & 80.66 & 49.67 & 33.36 & 29.76 & 28.09 \\
\hline 8 & 205.52 & 164.71 & 135.62 & 104.91 & 89.86 & & & $29.89-$ & 28.09 \\
\hline
\end{tabular}

Fig. 9. Scheme of calculating the minimal value of the objective function using random search by the best attempt based on the fragment of the table in Fig. 7.

The maximal prediction error of the interval model developed for this "saturated block" is 133,64 (denoted by ellipse in Fig. 5). According to expression (14), it is necessary to define the vector for which the maximal value of the objective function is found.

In the next iteration of the algorithm realization, the "contenders" are two "saturated blocks": $\psi(7,20)$ and $\psi(13,20)$, respectively:

$$
\begin{gathered}
\left\{\begin{array}{c}
47,492 \leq 19 b_{0}+2,944439 b_{1} \leq 58,037 \\
126,429 \leq 60 b_{0}+4,094345 b_{1} \leq 154,523
\end{array},\right. \\
\left\{\begin{array}{c}
78,901 \leq 35 b_{0}+3,555348 b_{1} \leq 96,456 \\
126,429 \leq 60 b_{0}+4,094345 b_{1} \leq 154,523
\end{array} .\right.
\end{gathered}
$$

The maximal prediction errors of interval model, the parameter areas of which were calculated from the solving of ISLAEs (20) and (21) are 28,09 and 28,09 respectively. The values of the maximal errors are denoted by ellipse in Fig. 10.

Thus, both ISLAE (20) and ISLAE (21), which provide the numerical value of the objective function 28,09 as a minimum of maximal error for interval models, can be considered the optimal "saturated block".

As we can see, it was necessary to carry out only 3 iterations to select the optimal "saturated block" by using the proposed method. These iterations correspond to the analysis of 3 variants of "saturated blocks".

Let us study the effectiveness of the method of forming a set of basic equations in the problem of ISLAE solutions localization based on a "saturated block". The proposed method, method of full combinatorial enumeration and random search methods are compared. To do this, it is necessary to define the most complex operation for finding the objective function. This is the calculation of inverse matrix for ISLAE at each stage of the "saturated block" computation.

Thus, in the example, for the same initial conditions, to define the set of basic equations, it was necessary to carry out the procedure of matrix inversion 190 times using the method of full combinatorial enumeration; 13 times using the method of random search with linear tactics; 23 times using the method of random search by the best attempt. Applying the new method proposed in this paper, it was necessary to carry out matrix inversion only 3 times.

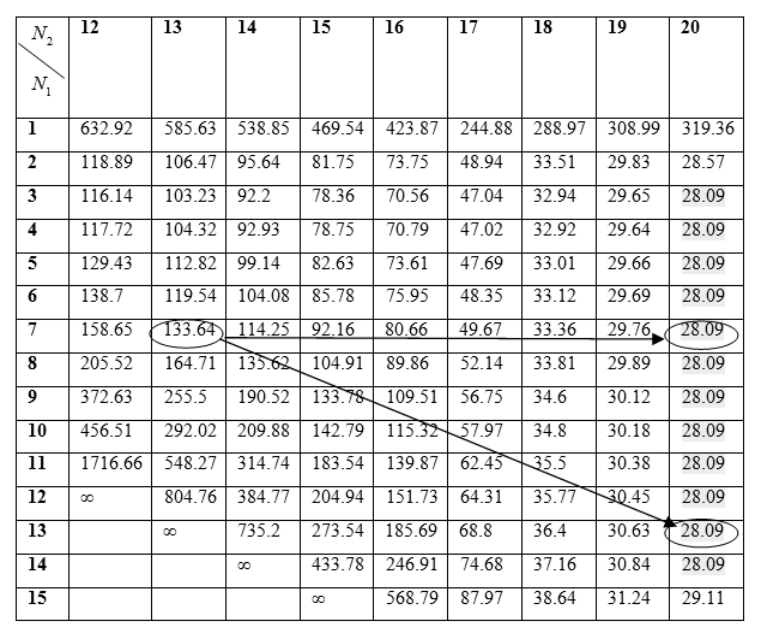

Fig. 10. Scheme of calculating of minimum value of objective function by the proposed method based on the fragment of table in Fig.7.

Therefore, by the criterion of computational complexity the proposed method for the given test example is 63 times more efficient than the full combinatorial enumeration method; 4 times more efficient than the method of random search with linear tactics; almost 8 times more efficient than the method of random search by best attempt. It should be noted that the considered problem has a small dimensionality. However, increasing the dimensionality of the problem leads to increasing the effectiveness of the proposed method.

\section{Conclusions}

The method of forming a set of basic equations in the problem of localization of ISLAE solutions based on a "saturated block" is proposed. The proposed method, unlike the existing ones, is based on solving the optimization problem with the criterion of minimization of maximal prediction error for interval models. The area of parameter estimations of these models is localized by the ISLAE solutions area in the form of a "saturated block".

A comparative analysis of the methods of random search of optimal "saturated block" and the proposed one in terms of their effectiveness is conducted. Its significant advantage by the criterion of computational complexity is represented. In the test example, it is shown that by the criterion of computational complexity the proposed method is 3 times more effective than that of random search with linear tactics.

\section{References}

[1] M. Dyvak, Tasks of mathematical modeling the static systems with interval data. Ternopil, Ukraine, 2011. (Ukrainian) 
[2] G. Alefeld and J. Herzberger, Introduction to interval computations, Computer Science and Applied Mathematics. New York, USA: Academic Press, Inc. Harcourt Brace Jovanovich Publishers, 1983.

[3] S.P. Shary, Algebraic Approach to the Interval Linear Static Identification, Tolerance, and Control Problems, or One More Application of Kaucher Arithmetic, Reliable Computing, vol. 2, no. 1, pp. 3-33, 1996.

[4] M. Dyvak, V. Manzhula and O. Kozak, "New method tolerance estimation of the parameters set of interval model based on saturated block of ISLAE", in Proc. IX-th International Conference CADSM'2007, pp. 376-379, Lviv-Polyana, Ukraine, 2007.

[5] L. Rastrigin, Adaptation of complex system. Riga, Latvia: Zinatne, 1981. (Russian)

[6] L. Rastrigin, A random search. Moscow, Russia: Znanie, 1979. (Russian)

[7] L. Rastrigin, Theory and application of random search, Institute of electronics and computers equipment, Riga, Latvia, 1969. (Russian)

[8] L. Rastrigin, Modern principles of management of complex objects. Moscow, Russia: Owls. radio, 1980. (Russian)

[9] E. Walter and L. Pronzato, Identification of parametric model from experimental data, London, Berlin, Heidelberg, New York, Paris, Tokyo: Springer, 1997, $413 \mathrm{p}$.

[10] C. F. J. Wu and M. S. Hamada, Experiments: Planning, Analysis and Optimization, Wiley, 2009.

[11] M. Dyvak, I. Oliynyk, and P. Stakhiv, "Method of reduction for interval system of linear algebraic equations and its application to modeling of the electric power generated by a small hydroelectric power station", in Proc. 17th International Conference on Computational Problems of Electrical Engineering, CPEE' 2016, Sandomierz, Poland, 2016.

[12] M. Dyvak and I. Oliynyk, "Method of formation of an optimal "saturated block" in the task of localization of solutions to interval system of linear algebraic equations", Inductive Modeling of Complex System, no. 8, pp. 79-99, 2016. (Ukrainian)

[13] M. Dyvak, I. Oliynyk, V. Manzhula, and R. Shevchuk, "Stochastic method of forming an optimal "saturated block" in the localization task of solutions to interval system of linear algebraic equations", in Proc. 14th International Conference CADSM (The Experience of Designing and Application of CAD Systems in Microelectronics), pp. 367-371, Lviv, Ukraine, 2017.

\section{МЕТОД ОЦІНЮВАННЯ МНОЖИНИ РОЗВ' ЯЗКІВ ІНТЕРВАЛЬНОЇ СИСТЕМИ ЛІНІЙНИХ АЛГЕБРИЧНИХ РІВНЯНЬ 3 ОПТИМІЗОВАНОЮ ПРОЦЕДУРОЮ ВИБОРУ "НАСИЧЕНОГО БЛОКУ"}

\author{
Микола Дивак, Ірина Олійник
}

Обгрунтовано необхідність застосування нового методу формування набору базових рівнянь у задачі локалізації розв'язків інтервальної системи лінійних алгебричних рівнянь (ІСЛАР) на основі “насиченого блоку”, який грунтується на розв' язуванні оптимізаційної задачі.

За критерій обрано мінімізацію максимальної похибки прогнозування інтервальними моделями, параметри яких належать області локалізації розв' язків ІСЛАР.

Проведено порівняльний аналіз ефективності запропонованого методу пошуку оптимального "насиченого блоку”, порівняно із методами стохастичного пошуку, зокрема, з лінійною тактикою та за найкращою спробою. Показано його суттєву перевагу за критерієм мінімуму обчислювальної складності.

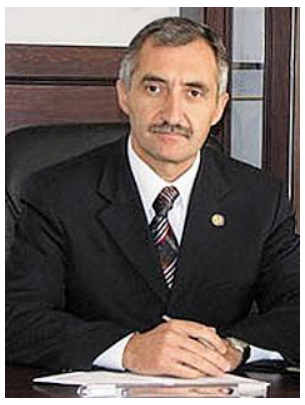

Mykola Dyvak - Professor (2004), D.Sc in Mathematical Modelling and Computing Methods (awarded by Lviv Polytechnic National University, Lviv, Ukraine (2003)). Post Ph.D. study in Intelligent Control, Tsinghua University, Beijing, China (1994). Ph.D. in Control of Technical System (awarded by Moscow Power Energy University, Moscow, Russia (1992)).

Currently, Dean of Faculty of Computer and Information Technologies at Ternopil National Economic University, Ukraine. Areas of research interest: mathematical modelling of static and dynamic systems, computing methods, interval data analysis, intelligent optimisation techniques.

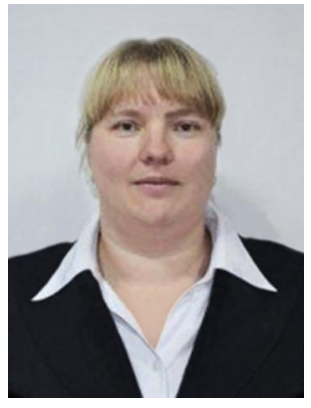

Iryna Oliynyk - Master's Degree in Computer Science, Ternopil National Economic University, Ukraine (2008). Currently, Ph.D student of the Computer Science Department, Ternopil National Economic University, Ukraine. Areas of research interest: mathematical modelling, stochastic optimization methods, interval data analysis. 